\title{
Fire-Net: A Deep Learning Framework for Active Forest Fire Detection
}

\author{
Seyd Teymoor Seydi, ${ }^{1}$ Vahideh Saeidi $\mathbb{D}^{2},{ }^{2}$ Bahareh Kalantar $\mathbb{D}^{\circ},{ }^{3}$ Naonori Ueda, ${ }^{3}$ \\ and Alfian Abdul Halin (iD) \\ ${ }^{1}$ School of Surveying and Geospatial Engineering, College of Engineering, University of Tehran, Tehran 14174-66191, Iran \\ ${ }^{2}$ Department of Mapping and Surveying, Darya Tarsim Consulting Engineers Co. Ltd, Tehran 15119-43943, Iran \\ ${ }^{3}$ RIKEN Center for Advanced Intelligence Project, Goal-Oriented Technology Research Group, Disaster Resilience Science Team, \\ Tokyo 103-0027, Japan \\ ${ }^{4}$ Department of Multimedia, Faculty of Computer Science and Information Technology Universiti Putra Malaysia, Serdang, \\ Seri Kembangan 43400, Malaysia
}

Correspondence should be addressed to Bahareh Kalantar; bahareh.kalantar@riken.jp

Received 31 August 2021; Revised 6 January 2022; Accepted 26 January 2022; Published 21 February 2022

Academic Editor: Giorgio Pennazza

Copyright (c) 2022 Seyd Teymoor Seydi et al. This is an open access article distributed under the Creative Commons Attribution License, which permits unrestricted use, distribution, and reproduction in any medium, provided the original work is properly cited.

\begin{abstract}
Forest conservation is crucial for the maintenance of a healthy and thriving ecosystem. The field of remote sensing (RS) has been integral with the wide adoption of computer vision and sensor technologies for forest land observation. One critical area of interest is the detection of active forest fires. A forest fire, which occurs naturally or manually induced, can quickly sweep through vast amounts of land, leaving behind unfathomable damage and loss of lives. Automatic detection of active forest fires (and burning biomass) is hence an important area to pursue to avoid unwanted catastrophes. Early fire detection can also be useful for decision makers to plan mitigation strategies as well as extinguishing efforts. In this paper, we present a deep learning framework called Fire-Net, that is trained on Landsat- 8 imagery for the detection of active fires and burning biomass. Specifically, we fuse the optical (Red, Green, and Blue) and thermal modalities from the images for a more effective representation. In addition, our network leverages the residual convolution and separable convolution blocks, enabling deeper features from coarse datasets to be extracted. Experimental results show an overall accuracy of $97.35 \%$, while also being able to robustly detect small active fires. The imagery for this study is taken from Australian and North American forests regions, the Amazon rainforest, Central Africa and Chernobyl (Ukraine), where forest fires are actively reported.
\end{abstract}

\section{Introduction}

Forests, lands dominated by trees, cover approximately 4 billion hectares of the earth's land area [1]. This is equivalent to around $29 \%$ of the earth. Forest management and conservation are therefore essential tasks for sustaining biodiversity at global scale [2]. Forest fires, which can be manmade or naturally occurring, are events that can threaten our planet leaving behind catastrophic circumstances such as damages and losses [3-7]. Forest fires have long-term devastating effects on ecosystems such as destroying vegetation dynam- ics, emission of greenhouse gases, loss of wildlife habit, and also devastation of land covers [8-11]. Therefore, accurate and timely detection of active forest fires is critical to minimize and/or prevent such hazards.

In recent years, advanced remote sensing (RS) technologies have been adopted to monitor and observe the earth and land covers, which can be on a large scale [12-15]. Availability of high spatio-temporal resolution data and multispectral imagery allow tremendous applications of forest monitoring such as burned area mapping [7], active fire detection [11, 16], burning biomass detection [17, 18], and forest 
disturbance monitoring $[19,20]$. It is worth noting that one widely and freely available RS data source can be used for all the tasks, which are imagery form Landsat satellites.

Active fire detection is an important application of RS in forest monitoring. The active fire detection and analysis can be used in many applications such as source point pollution in air quality analysis, initial seed point in burned area mapping, and prediction of growth and spread of active fires [21]. Until now, many researchers detect active fires using multispectral satellite imagery, assisted by computer algorithms. For instance, Wooster and Nightingale [4] presented an active fire detection method based on Sentinel-3 imagery using Land Surface Temperature Radiometer (LSTR) near nadir scans and fire characterization (potential fire pixel classification) through middle infrared radiance calculation method. Cruz et al. [22] proposed a fire detection method according to color index. Their method was based on classification of vegetation to detect the smoke and fire flames. They claimed their method could be applied in real-time using unnamed aerial vehicle (UAV) dataset. Schroeder et al. [23] studied active fire detection by exploiting Visible Infrared Imaging Radiometer Suite (VIIRS) sensor data $375(\mathrm{~m})$. Their method deployed a contextual algorithm built on the heritage MODIS (MODerate Resolution Imaging Spectroradiometer) fire and thermal anomaly product. Jang et al. [24] designed forest fire detection algorithm based on Himawari-8 geostationary satellite data for detection of fire candidate pixels with thresholding based on multitemporal analysis. Jiao et al. [25] proposed a deep learning based forest detection algorithm based on UAV dataset. They used YOLOV3 (You Only Look Once version 3), RGB (Red, Green, and Blue) imagery. They reported the potential of YOLOV3 in detection of location of active fires. Yuan et al. [26] designed an active fire detection framework based on UAV imagery for forest areas. Their method was deployed in three stages: (1) color detection for forest candidate pixels, (2) motion detection for reducing false alarm pixels, and (3) classification fires pixels based on extracted features (direction and magnitude). Schroeder et al. [21] detected the active fire by using Landsat- 8 imagery for both daytime and nighttime fires. They applied thresholding short-wave infrared, optical and near infrared bands. Amraoui et al. [27] presented the active fire detection based on Meteosat-8/SEVIRI (Spinning Enhanced Visible and Infrared Imager) dataset. The fire detection in this framework as following: (1) mask non-target object (water, desert, urban), (2) identification high potential pixels based on thresholding brightness temperature and solar zenith angle, (3) removing contaminated pixels (cloud pixels, sun glint, highly reflective surfaces) by thresholding spectral and thermal bands, and (4) making decision on obtained potential fire pixels by obtained contextual information. Chen et al. [28] proposed a Convolutional Neural Network (CNN) based forest fire detection method using UAV dataset. In their framework after dataset capturing, some preprocessing such as histogram equalization, low-pass filtering was conducted and then, the CNN network with seven convolution layers and some hidden layers were utilized for binary classification. Gargiulo et al. [29] designed a fusion framework for active fire detection using Sentinel-2 imagery and CNN to improve the spatial resolution Short Wave Infrared (SWIR) bands toward $10 \mathrm{~m}$ resolution. They enhanced the spatial resolution of SWIR bands and improved the performance of active fire detection. Lin et al. [30] proposed an active fire detection framework using FengYun-2G dataset. The active fire detection procedure in this framework was based on comparing predicted value and observed value. That algorithm deployed image analysis for reducing false alarm pixels and temporal analysis for confirming fire pixels. Zhang et al. [31] investigated the active fire detection based on VIIRS dataset. This method was applied in 5 steps: (1) initial data screening by thresholding spectral and thermal bands, (2) data partitioning, (3) the detection of thermal anomaly pixels, (4) contextual analysis, (5) confirmed thermal anomaly pixels based on thresholding of the view zenith and solar zenith angles.

Similarly, there are many active fire detection frameworks exploiting RS datasets. In general, active fire detection methods based on traditional fire detection methods can be applied based three basic principles [32]: (1) detection of hot-temperature pixels using thresholding methods, (2) context-based methods where hot-temperature pixels are compared with the background, and (3) detection of fire pixels based on generated smoke and moving fire plumes. Generally, these active detection methods using RS imagery face their own respective challenges. Thresholding methods tend to fail due to tremendously varying environmental conditions and air temperature. Air temperature variations originating from various factors such as shadow, clouds, wind, illumination angle, seasonal variation, and climate change, can also be a problem for contextual methods. Then, small fires make smoke and non-fire objects detection problematic, especially when dealing with medium and lowresolution RS dataset (i.e. VIRIIS, and MODIS, Sentinel-3). It is also worth mentioning that most of the aforementioned methods rely on traditional machine learning classification, which requires feature engineering.

As one of the widely used RS data, Landsat- 8 satellite data was selected for this research. The dataset has higher spatial resolution compared to other modalities such as MODIS, VIRIIS, and Sentinel-3. Landsat- 8 also allows improved affordability in detecting small fires as the high-resolution imagery allows for a potentially more improved discrimination capabilities. To minimize the challenges mentioned above (pertaining to datasets), it is necessary to design an advance method for active fire detection. Therefore, this research presents a novel active fire detection method based on deep learning. This method combines the RGB and Thermal images from Landsat- 8 satellite imagery. The proposed deep learning framework has two-stream channels and can detect deep features by exploiting the prowess of its convolutional neural network architecture. The key contributions of this research are presented as follows:

(a) Presenting a framework for active fire detection based on deep learning called active-fire-net (Fire-Net).

(b) High efficiency against detection of small fires using freely available data 
(c) Taking advantages of deep features that combined spatial and spectral features instead of single spectral features

(d) Accurate and automatic detection of active fires with the potential of being applied as real time

(e) Taking advantages of residual and depth-wise and separable convolution block

(f) Introducing novel loss function for imbalancing problems

(g) Testing the transferability of training datasets

\section{Study area}

Table 1 describes the five study areas selected for active fire detection. Generally, the areas cover North America, Australia, the Amazon rainforest, Central Africa and Chernobyl (Ukraine). Different areas were selected due to the varying climate conditions, temperature affecting the thermal bands and also forest density. This allows for a variety of data to be analyzed with the hope of producing a more generalized model. The Fire-Net model utilizes training data from the active fires in North America and Australia. Model testing is done on the Central Africa, Brazil, Chernobyl and also data from Australia (the parts were not involved in training). [33]. The North American's forest includes 4 regions (Figure 1(a)) where the active fires were reported in 20132014. The Australian forests, which had average temperature of $19^{\circ} \mathrm{C}$ and humidity of $62 \%$, were obtained in June 2019 where the southern and eastern parts of Australia exhibited intense wildfire. The area includes 6 sub-regions with active fires in 2019-2020 (Figure 1(b)). The third study area is part of the Amazon rainforest, whose average humidity and temperature are $76 \%$ and $18.5^{\circ} \mathrm{C}$, respectively. The fourth study area is that of north-central Africa wildfires (occurred in December 2018), which was caused by human activity. The average of humidity and temperature of this study area are $72 \%$ and $25^{\circ} \mathrm{C}$, respectively. The fifth study area belongs to Chernobyl, Ukraine. The wildfires began in April 2020, which was spread quickly by wind. At the time of wildfire, the scene was mostly cloudy. Due to variations temperature and presence of cloud, this case study was chosen to evaluate the performance of proposed methodology against mentioned factors. The average of humidity and temperature are $46 \%$ and $9^{\circ} \mathrm{C}$, respectively.

2.1. Landsat-8 Dataset. The Landsat- 8 satellite sensor was designed by NASA (National Aeronautics and Space Administration) and was launched on into orbit in February 2013. This sensor is able to capture data in 11 spectral bands with spatial resolutions of 15 meters (panchromatic), 30 meters (visible, NIR, and SWIR), and 100 meters (thermal). Furthermore, this sensor collects dataset form Earth with a temporal resolution of 16-days. Since this study requires 4 spectral bands (R, G, B, and thermal), we chose the standard terrain corrected Level $1 \mathrm{~T}$ datasets.
2.2. Inventory data. The inventory (sample) data is meant as training images (maps and images) for the proposed supervised Fire-net network. The quality and quantity of sample data are the most important factors to obtain promising result. Therefore, we carefully identified the location of active fires from related resources such as [34, 35]. This was mainly done through visual inspection guided by expert knowledge to generate the reference data. The corresponding active fire locations were then extracted from Landsat8 datasets for training (65\% of the samples), validating (15\% of the samples), and testing ( $20 \%$ of the samples). In this research 722 patches were generated with the size of $256 \times 256$ pixels representing the training, validation, and testing datasets by 469,109, and 144 patches, respectively.

\section{Methodology}

Out of five study areas, two regions were selected as the training and validation samples for the Fire-Net deep learning algorithm and multi-scale residual learning networks (MSR-U-Net). This two-streamed pixel classification deep learning-based (Fire-Net) method was proposed to detect active fires from Landsat- 8 imagery. This network uses the encoder-decoder architecture for active fire detection. Due to the unique structure of active fires and spatial resolution of the Landsat- 8 dataset, a two-stream deep feature extractor architecture is proposed. This means that the Fire-Net framework consists of two deep feature extractor channels. The first channel is meant to detect the active fires whereas the second channel for background and non-fire objects detection. The general overview of Fire-Net is presented in Figure 2.

For dataset preparation, the Landsat- 8 images are preprocessed and converted into image patches. Image patches can suppress speckle noise and optimize segmentation. Basically, we divide each image into a grid of patches, specifically, $256 \times 256$ in size. Since we are considering 4-bands, the total number of $256 \times 256$ images are 4 . As with any other machine learning algorithm, we divide the dataset into three parts: (1) training dataset, (2) validation dataset, and (3) testing dataset. The training and validation datasets (which form what is called the development set) were used to discover the optimal weights for the entire network. For this work, we defined the stopping condition as 250epochs. The testing dataset was used to evaluate the trained-and-validated network where seven metrics were calculated. Finally, the trained network is deployed to detect the active fires through the second (Australian Wildfire, the parts were not involved in training), third (Amazon rainforest), forth (Central Africa), and fifth study areas (Chernobyl). The next subsection presents the details of Fire-Net deep learning architectures.

3.1. Image Pre-processing. For the landsat-8 L1-T product some pre-processing as radiometric correction and orthorectification were conducted. The atmospheric correction was also done by Fast Line-of-sight Atmospheric Analysis of Hypercubes (FLAASH) modules. Besides, the thermal bands need to convert into radiance and then to brightness 
TABLE 1: The descriptions of datasets for five study areas.

\begin{tabular}{lcc}
\hline Case study & Date & Description \\
\hline North American & $2013-08-31,2014-09-19$ & Training, and validation \\
Australian wildfire & $2019-09-03,2019-10-19,2019-10-21,2019-10-28,2019-11-06$, & Training, validation, and testing \\
Amazon rainforest & $2019-11-13,2019-12-08,2019-12-15,2019-12-31,2020-02-01$ & Testing the transferability \\
Central Africa & $2019-08-25$ & Testing the transferability \\
Chernobyl & $2019-01-04,2018-12-19$, and 2018-12-19 (different area) & Testing the transferability \\
\hline
\end{tabular}

temperature. The final step was splitting data to small patches with size of $256 \times 256$ pixels to be fed into Fire-Net.

\subsection{Proposed Deep Learning Architecture (Fire-Net). A two-} stream feature deep learning method was proposed for forest fire detection. This architecture is based on multiscaleresidual convolution layers where it takes the advantage of the point/depth-wise convolution, residual, and multiscale convolution blocks. The multiscale block increases the robustness of Fire-Net against size variations [36]. This allows Fire-Net to improve detection performance of small fires. The residual blocks also has been shown to prevent the vanishing gradient problem and also affords efficiency equal to that of a deeper network [37]. The depth/point-wise convolutions furthermore are cheaper operators that effectively reduce the number of model parameters and computational cost [38]. The Fire-Net architecture is presented by Figure 3 in two-streams for deep feature extraction. Firstly, the shallow features are extracted by the multiscale $2 \mathrm{D}$ convolutional layers. Next, the extracted deep features are fed to each deep feature extractor channels. Since small fires can cover small areas, we used up-sampling with a rate of two for the first channel before extracting the deep features. This stream combined residual block and multiscale residual block for extracting deep features. Simultaneously, the second channel was discovered by the deep features from the original resolution dataset by combing the residual block and multiscale residual block that are presented by Figure 4 . Then, the extracted deep features from two layers were fused by the summation operator. Finally, the extracted features were fed to $2 \mathrm{D}$-convlotution layer as a single feature map to decide on the final class label (active fire or not). The details of Fire-Net architecture are explained in next subsections.

3.2.1. Convolution Layers. In $\mathrm{CNN}$, the main task of the convolution layers is to extract high level deep features from the input dataset $[39,40]$. For a convolutional layer in the 1-th layer, the computation is expressed according to equation (1) $[41]$.

$$
y^{l}=g\left(w^{l} x^{l-1}\right)+b^{l}
$$

where $x$ is the input data from layer $l-1 ; g$ is the activation function; $w$ is the weighted template; and $b$ is the bias vector.

In $2 \mathrm{D}$ convolution layer, the output of the $j^{\text {th }}$ feature map $(f)$ in $i^{\text {th }}$ layer at the spatial location $(x, y)$ can be com- puted using equation (2) [41].

$$
f_{i, j}^{x y}=g\left(b_{i, j}+\sum_{m} \sum_{r=0}^{R_{i}-1} \sum_{s=0}^{s_{i}-1} W_{i, j}^{r, s} v_{i-1, m}^{(x+r)(y+s)}\right)
$$

where $m$ is the feature cube connected to the current feature cube in the $(i-1)^{\text {th }}$ layer; $W$ is the $(r, s)^{\text {th }}$ value of the kernel connected to the $m^{\text {th }}$ feature cube in the preceding layer; and $R$, and $S$ are the length and width of the convolution kernel size, respectively.

The Fire-Net architecture employs three strategies: (1) consistent with [36], we make use of multiscale kernel convolution (i.e. different kernel size convolutions) to ensure robustness against variations in scale [42], (2) we prevent the problem of vanishing or exploding gradient by utilizing residual blocks (or skip connection) layers, which allows the gradient to be directly back-propagated to earlier layers [43], and (3) Depth/Point-wise convolution block: this kind of convolution layer considers only a single filter for each input feature $[44,45]$. Figure 5 represents the main differences of standard and depth/point-wise convolution layers.

The loss function used for measuring the training error is based on predicted value of true value [46]. This research is used a hybrid loss function (Equation (3)) is known weightbinary-cross-entropy-dice (WBCED) loss function that it is more efficient for small targets [47]. The WBCED loss function is combined dice loss (Equation (4)) and weighted cross entropy loss (Equation (5)) that can be defined between predicted value $(p)$ of true value $(y)$ as following:

$$
\begin{aligned}
& \text { Loss }_{W B C E D}=\text { Loss }_{\text {weighted binary cross entropy }}+\text { Loss }_{\text {Dice }} \\
& \text { Loss }_{\text {Dice }}=1-\frac{2 \sum y \times p}{\sum y+\sum p} \\
& \text { Loss }_{\text {weighted binary cross entropy }}=-w(y \log (p)+(1-y) \log (1-p)) \\
& \begin{array}{c}
\text { Loss }_{\text {binary cross entropy }}=-(y \log (p)+(1-y) \log (1-p)) \\
w=\frac{\sum_{i=1}^{s} \sum_{j=1}^{\mathrm{t}} x_{i, j}}{e^{-5 \times \mid \text { Mask-0.5|} / \sum e^{-5 \times \mid \text { Mask-0.5 }}}}
\end{array}
\end{aligned}
$$

where Mask is obtained by overage pooling of reference map furthermore, $s, t$ are width and length of Mask.

The Fire-Net architecture is trained by an Adaptive Moment Estimation (Adam) optimizer [48] through 


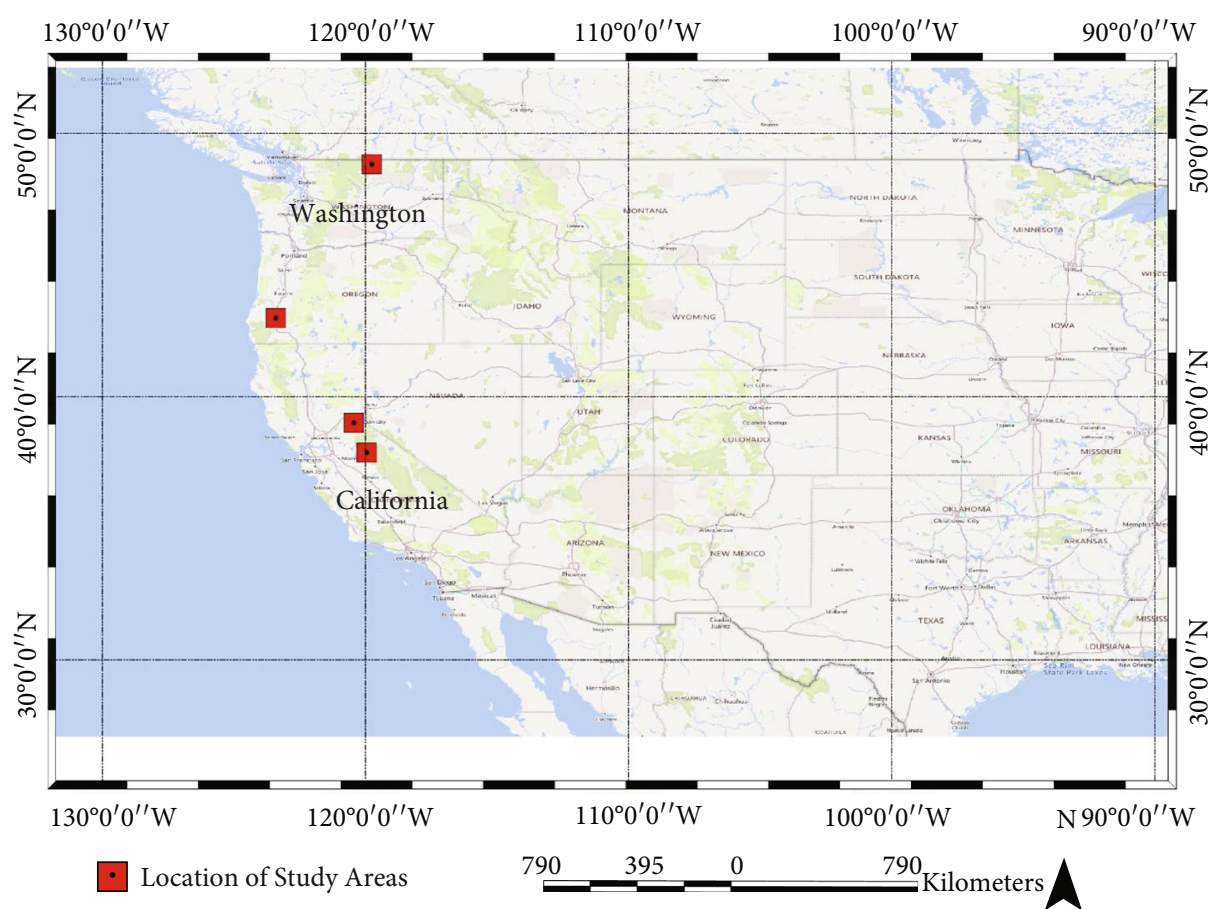

(a)

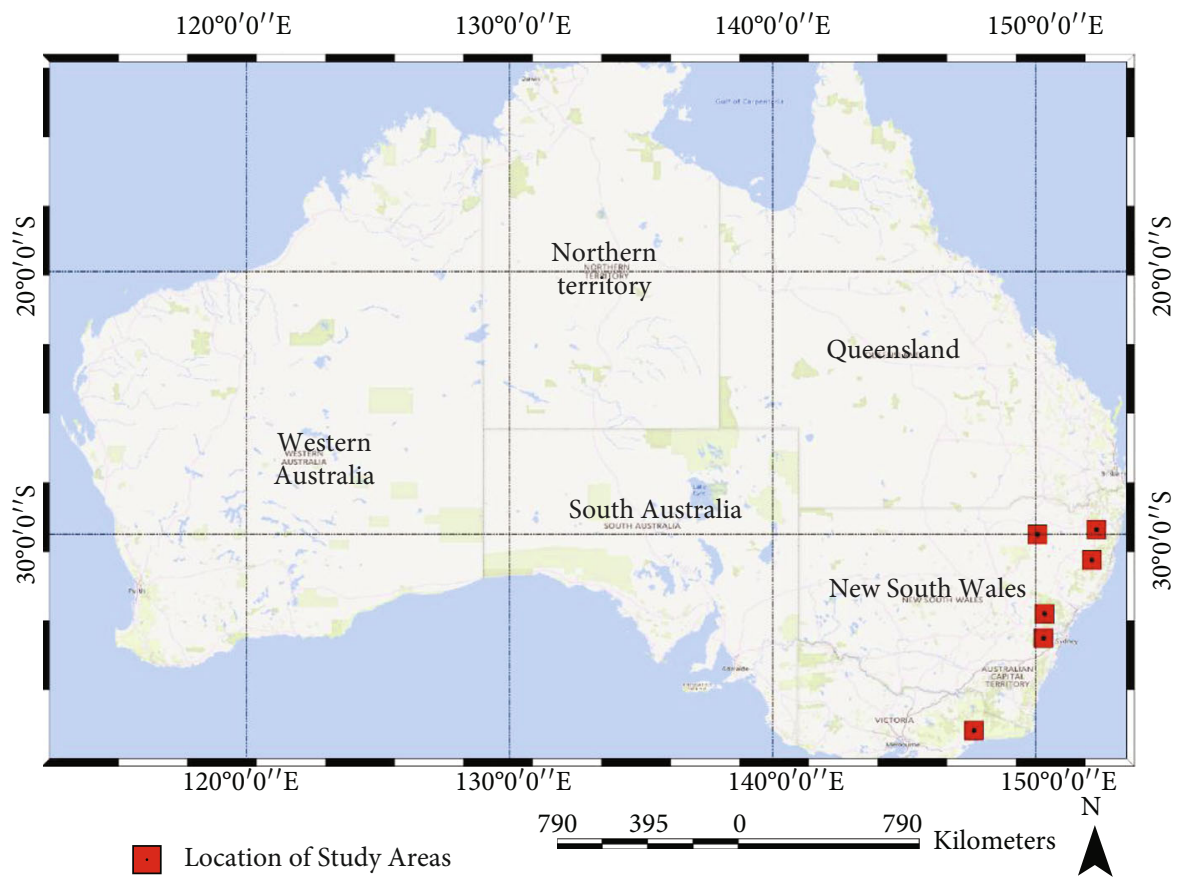

(b)

FIgURE 1: Location of active fire in (a) the USA, (b) Australia.

backpropagation where weights were initialized using the Golorot initializer [49]. The hyperparameters for training Fire-Net is as follows:

(i) Batch-size $=7$-patches

(ii) Learning rate $=10^{-3}$

(iii) Number of Epochs $=250$
The shuffle technique was used during training process. Fire-Net was implemented on Tensorflow v2.4.1 and Keras v2.4.3.

3.2.2. Evaluation Indices/methods or accuracy assessment. We performed two types of evaluation for Fire-Net. Firstly, visual analysis was performed where the results were compared with reference data. Next, we calculated seven quality 


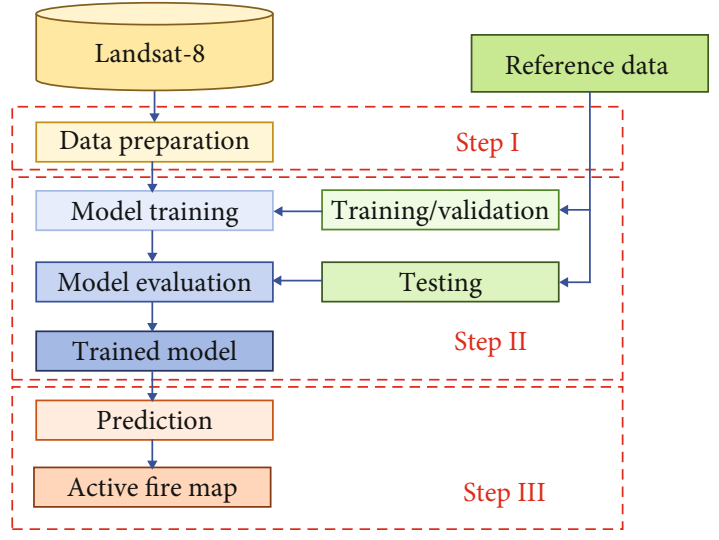

Figure 2: Overview of general framework of active fire detection.

indices namely overall accuracy $(\mathrm{OA})$, recall, precision false positive rate (FPR), miss-detection (MD), F1-score, and kappa coefficient (KC). More details of these indices can be found in [41]. We also performed a comparison between Fire-Net with an established CNN, namely the MSR-UNet. The main reason is due to MSR-U-Net's similarity to Fire-Net in combining multi-scale kernel convolution filters with residual blocks deep features extraction [50, 51]. MSRU-Net also uses an encoder-decoder structure for exploring deep features. For a detailed evaluation of Fire-Net's efficiency, 5 machine learning methods (K-Nears-Neighbor (KNN), Support Vector Machine (SVM), Multi-Layer Perceptron (MLP), Random Forest (RF), and Extreme Gradient Boosting (XGBoost)) were used for comparing fire detection results. Brief explanations of each algorithm are provided in the following:

(1) KNN: This is a simple non-parametric classifier. Without any assumption about the dataset, KNN classifies the data based on each data point's neighbors. This research used the Euclidian metric and the numbers of neighbors were set to 4

(2) SVM: The SVM is a supervised machine learning classifier that is widely used in RS. The main idea behind SVM is to find a hyperplane that best separates a dataset into two-classes. Although in its original form, the SVM is meant for binary classification, it can still be used for multi-class classification tasks. In general, a kernel function is used to generate a nonlinear decision boundary in the feature space. In this work, we empirically tuned two of the SVM parameters, which are the penalty coefficient and the (Radial Basis) kernel parameter. They were set to $10^{1}$ and $10^{3}$, respectively

(3) MLP: This classifier is a supplement of a feed forward neural network. The MLP has several hidden layers where each has several neurons. The neurons are trained using the back propagation learning algorithm. We constructed a two hidden-layer MLP with 40 and 30 neurons in the first and second layers, respectively
(4) RF: RF is a supervised learning classifier consisting of an ensemble of decision trees where the final decision is obtained by majority voting. RF is trained by a bagging method where the gist of bagging is the combination of learning models that improves overall classification results. This algorithm has two main parameters i.e. number of trees and number of features to split. Each were set to 93 and 3, respectively

(5) XGBoost: XGBoost is a decision-tree-based ensemble supervised learning method, based on the gradient boosting framework. Several parameters need to be tuned for this classifier, namely number of estimators (set to 105), max-depth (set to 4), eta (set to 0.03 ), min-child-weight (set to 1 ), subsample (set to 0.8 ) and colsample-bytree (set to 0.8 ).

\section{Results}

This section explains the experimental setup and results of the proposed active fire detection algorithm (Fire-Net). The dataset we used contains 22 tiles of Landsat- 8 images covering the first and second study areas (i.e. North USA and Australia). From the 22 tiles, 14 tiles were used for training, 3 tiles for validation, and 5 tiles for testing. For the ease of processing, the 22 tiles were split into small patches in size of $256 \times 256$. At the end, to check the transferability of the Fire-Net method it was tested on other study areas (i.e. the Amazon rainforest, Central Africa and Chernobyl).

4.1. Australian Forest. The results of the Australian's forest fire detection, by methods, are presented by Figures 6(b) and $6(\mathrm{c})$. Visually, both deep learning methods managed to detect fire areas. However, misclassification occurred in the patch boundaries for MSR-U-Net (Figure 6(b)). Based on the evaluation metrics, the main difference between the two networks were in detection of active fires with low MD and FPR rates. Many false positive pixels were detected using MSR-U-Net, while Fire-Net had lower false positives.

The numerical results of active fire detection for the first test are presented in Table 2(a). Fire-Net outperformed MSR-U-Net with a higher overall accuracy of $99.95 \%$, precision of $97.94 \%$, F1-Score of $97.57 \%$, and KC of 0.975 indices. MSR-U-Net exhibited a lower rate of MD (near 0\%), while considerably showing a higher FPR compared to Fire-Net. There is however a trade-off between detecting fire pixels and non-fire pixels. The Fire-Net model has lower error rates for active fire detection whereas MSR-U-Net has lower MD rates but higher FPR rates. The ideal situation is when a method can effectively detect both fire and non-fire pixels with lowest error. The numerical results for active fire detection by the other machine learning algorithms are presented in Table 3(a). Although the SVM had the most accurate OA (99.03\%), its performances against the other indices of Precision, Recall and F1-Score were low. The MD index (99.98\%) for SVM revealed that it could not effectively detect active fires. RF and XGBOOST on the other hand showed good performance compared to the other algorithms. In all 


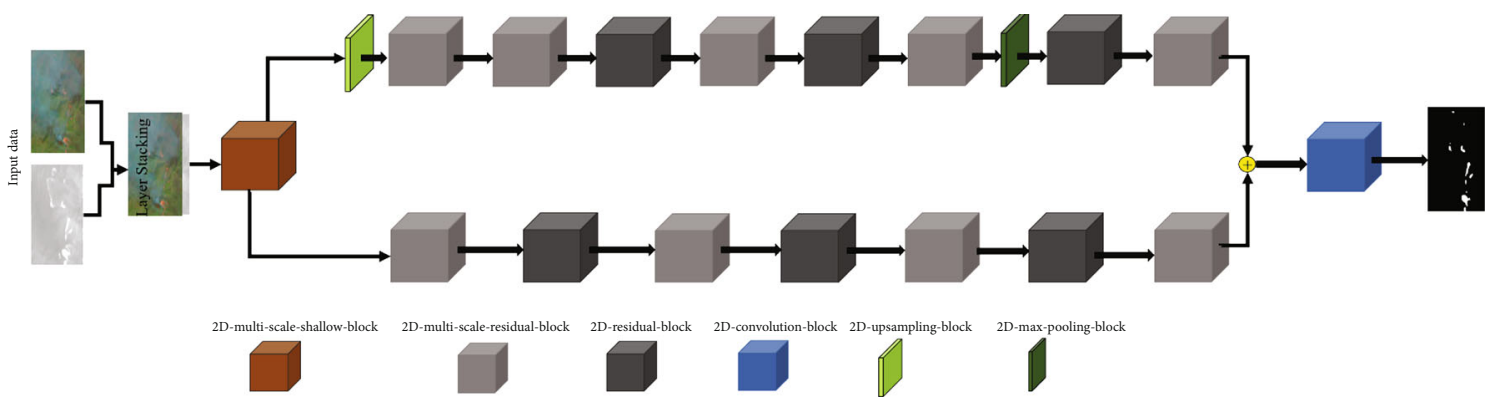

FIGURE 3: The training process for active fire detection.

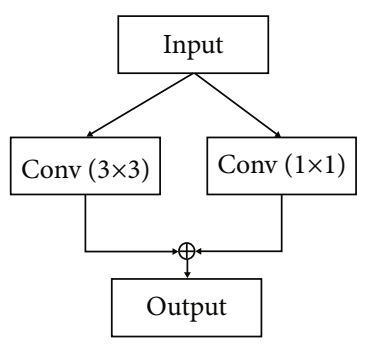

(a) Shallow Deep Feature Extractor

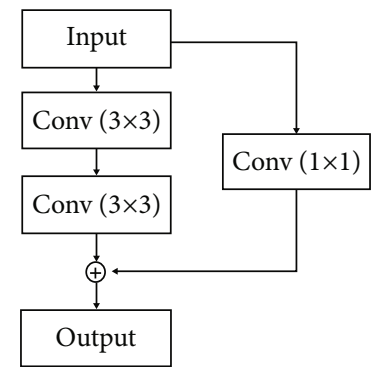

(b) Residual Block

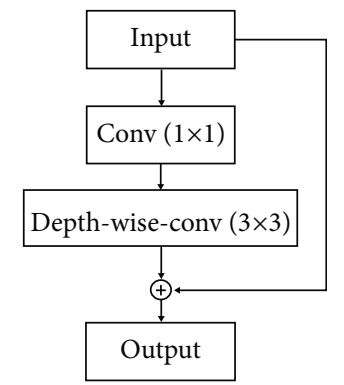

(c) Residual Point/Depth-Wise Block

FIgURE 4: Three convolution blocks in the Fire-Net architecture.

however, Fire-Net outperformed all these algorithms where they lacked the robustness, correctness, and certainty against the 7 evaluation metrics.

4.2. Central Africa Forest Fire. The result of active fire detection for Central Africa is presented in Figures 6(f) and 6(g). It shows background from foreground segmentation for each scene. For this case study, the active fire areas were small compared to the north of USA and Australia. Both methods were able to detect active fires with cases of misclassifications.

Fire pixels can be seen when zooming into Figures 7(b) and 7(c). Compared to MSR-U-Net, Fire-Net provides more fitting segmentations, covering more relevant areas of fire areas. This indicates that Fire-Net detects almost all relevant fire pixels that were not detected by MSR-U-Net.

Table 2(b) shows the accuracy assessments for both networks. The results indicate that both methods performed well in detecting non-fire pixels, while their accuracies decreased when detecting fire pixels. For this dataset, the $\mathrm{MD}$ rate is high and both networks focused on non-fire pixels. The low values of $\mathrm{KC}$ indicate the decrease of perfor- mance reliability of both networks. Table 3(b) shows the quantitative evaluations for 5 machine learning methods to detect active fires. Accordingly, most of them were successful in detection of non-active fires as indicated by FPR values lower than 1\%. Moreover, the lower Precision and F1Scores values show that consistency and balance among all 7 evaluation metrics were not seen amongst those 5 methods compared with Fire-Net.

4.3. Brazilian's Forest Fire. The active fire maps for Brazil's forest produced by the different methods are illustrated in Figures $6(\mathrm{j})$ and $6(\mathrm{k})$. The southern part of the region was mainly classified as having active fires. Seemingly, both methods generated active fires map with lowest error.

Figures $7(\mathrm{~g})$ to $7(\mathrm{i})$ presents a closer look of the active fire areas. Figure $7(\mathrm{i})$ is the active fire map generated by Fire-Net. It is comparable and as complete as the one produced by MSR-U-NET. The Accuracy assessment for the generated fires maps are presented in Table 2(c). It can be seen that both methods successfully detected the fires, with Fire-Net outperforming MSR-U-Net. Fire-Net's precision is 95.98\%, recall 98.04\%, near zero-FPR, and an F1-Score of 


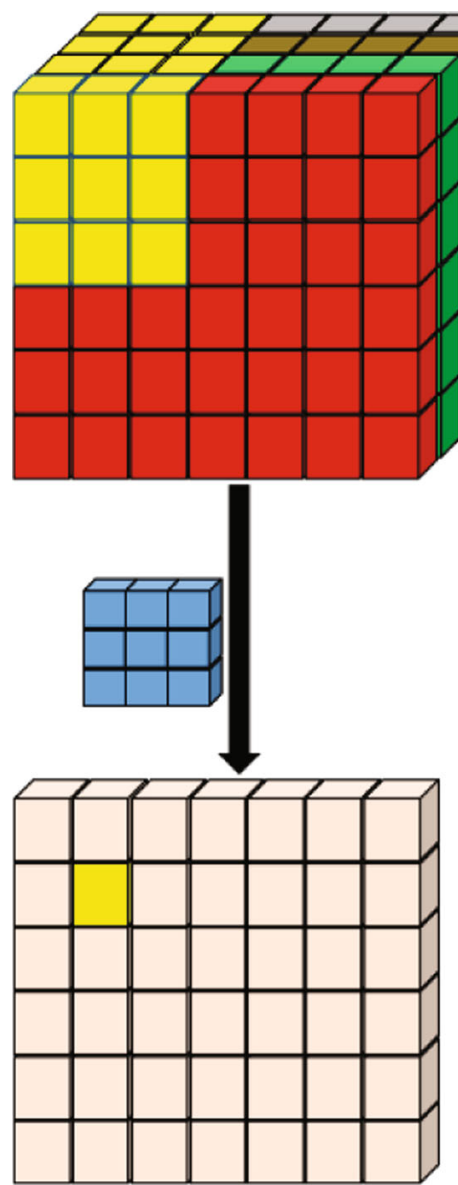

(a) Standard Convolution
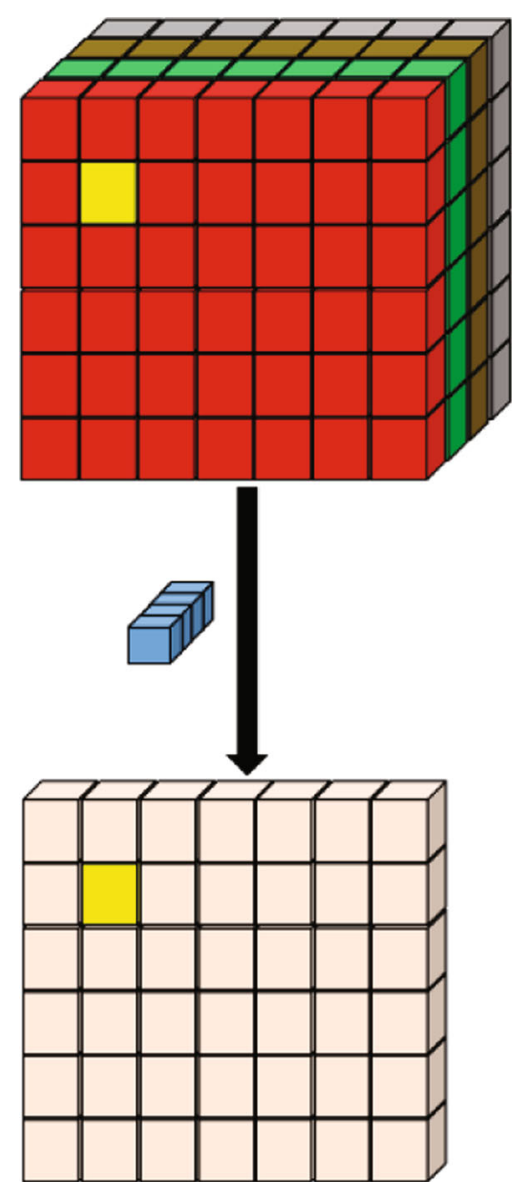

(b) Point-Wise Convolution

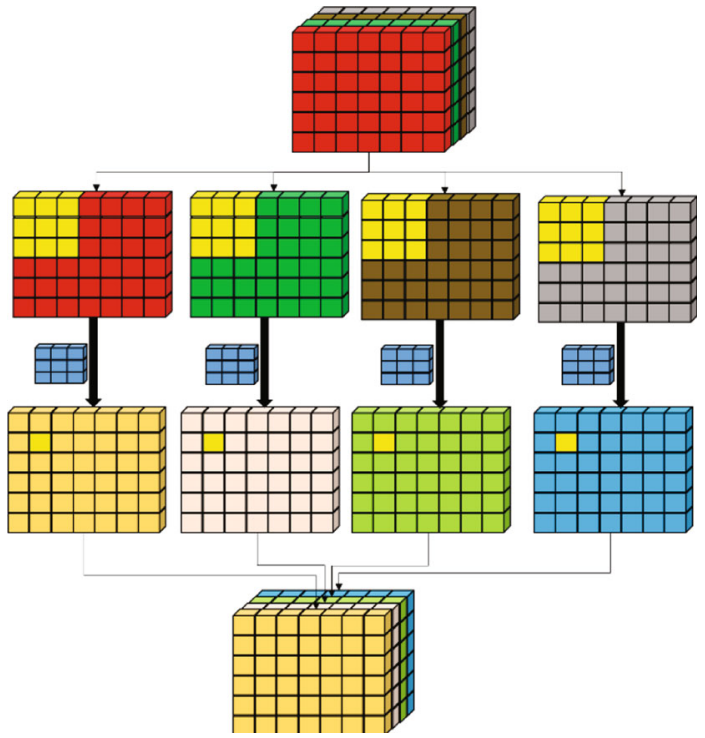

(c) Depth-Wise Convolution

Figure 5: Comparison of three convolution layers in the Fire-Net architecture.

97.0\%. The numerical assessments for the 5 machine learning algorithms are shown in Table 3(c). OA was mostly equal for all at approximately 99\%. Precision on the other hand were lower than $60 \%$ with low FPR rates (below 1\%) and higher MD rates. These indicate weak active fire detec- tion. Therefore, the 5 machine learning methods were not comparable with Fire-Net in this region.

4.4. Chernobyl's Forest Fire. Figures 6(n) and 6(o) shows both network's fire detection performance in the Chernobyl 


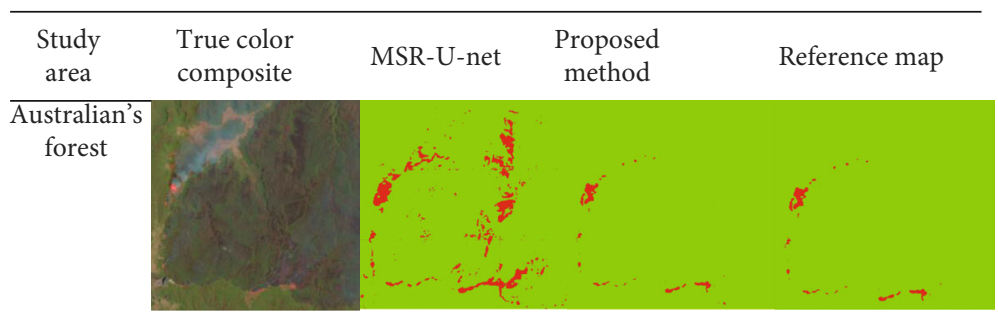

(a)

(b)

(c)

(d)

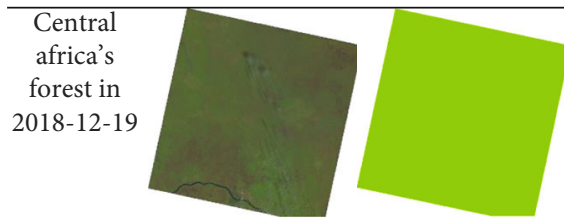

(e)

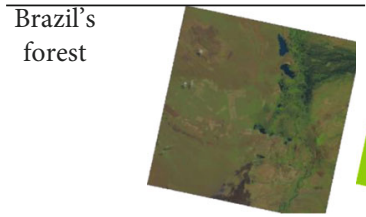

(i)

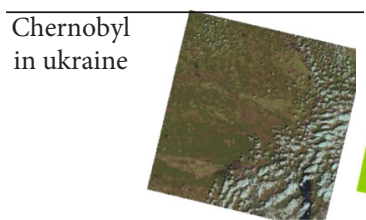

(m) (f)

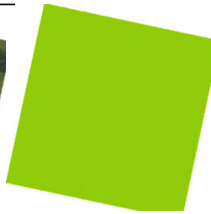

(j)

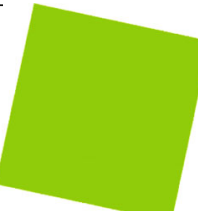

(n)

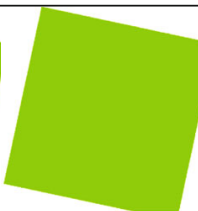

(g)

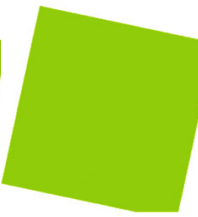

(k)

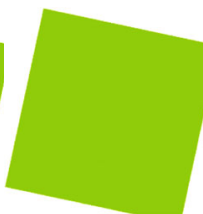

(o)

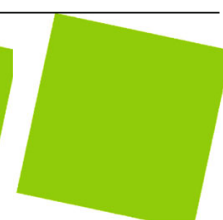

(h)

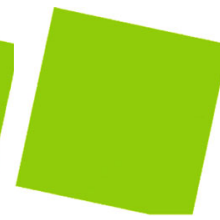

(l)

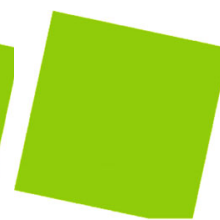

(p)

Figure 6: The result of active fire detection and red and green are fire and non-fire, respectively.

forest. Note that fire detection in this region is challenging due to the presence of high clouds and fires of small sizes. Nonetheless, both networks performed well with MSR-UNet failing to detect a small number of active fires (Figures $7(\mathrm{j})-7(\mathrm{~m}))$. As with the other regions, Fire-Net showed higher precision, recall, and f1-Score. However, we posit that the diversity of the terrain might be the cause for higher MD rates compared to FPR. The results of active fire detection using the 5 machine learning methods are presented in Table 3(d). OAs exceeding $99 \%$ for KNN, RF, and XBGOOST, with an added low FPR (below 1\%) indicates accurate active fire detection. However, SVM and MLP were successful in the mapping of non-active fires. Again, based on the 7 evaluation metrics, the Fire-Net method showed its superiority.

\section{Discussion}

5.1. Accuracy. Timely detection of active fires is critical for disaster management. This research proposes a deep network called Fire-Net for active forest fire detection based on optical RGB and thermal dataset. The accuracy of the network was evaluated based on 4 different case study areas. Furthermore, Fire-Net's results were also compared against the established MSR-U-Net deep neural network. The evaluation metrics and visual inspection of active fire detection results showed that the proposed method outperformed the MSR-U-Net and exhibited higher accuracy in detection of fire and non-fire pixels. For a more detailed analysis, Fire-Net was compared against 5 other popular machine learning algorithms. It was discovered that MLP, RF, SVM, $\mathrm{KNN}$, and XGBOOST required a relatively small number of samples, with the exception that several parameters must be tuned for optimal classification. Despite good performance, all these methods require manual handcrafted features which is laborious and time-consuming.

As for the evaluation metrics, Recall is an evaluation metric that finds true positive (TP) pixels. The mean of recall of the Fire-Net algorithm was $92.64 \%$ while MSR-U-Net was $86.25 \%$. This indicates Fire-Net's superiority in detecting more TP pixels. On the other hand, precision is a metric that describes a model's ability to identify positive pixels. In our study, Fire-Net had a precision of $93 \%$, indicating highconfidence in detection of active fire pixels. MSR-U-Net on the other hand only had a precision of under $67 \%$. Although Fire-Net clearly has higher precision and recall scores, the F1-Score is important to measure as well since it provides a harmonic mean of precision and recall. With regards to 
TABLe 2: Accuracy assessment of active fire detection (Comparison of Fire-Net and MSR-U-Net).

\begin{tabular}{|c|c|c|c|c|c|c|c|}
\hline Method & $\mathrm{OA}(\%)$ & Precision (\%) & Recall (\%) & FPR (\%) & MD (\%) & F1-score (\%) & $\mathrm{KC}$ \\
\hline \multicolumn{8}{|c|}{ (a) Australian's Forest } \\
\hline MSR-U-net & 94.73 & 16.55 & 100 & 4.91 & 0.00 & 28.40 & 0.272 \\
\hline Fire-net & 99.95 & 97.94 & 97.20 & 0.02 & 2.79 & 97.57 & 0.975 \\
\hline \multicolumn{8}{|c|}{ (b) Central Africa's Forest in 2018-12-19 } \\
\hline MSR-U-net & 99.99 & 72.91 & 70.70 & 0.0001 & 29.29 & 71.79 & 0.429 \\
\hline Fire-net & 99.99 & 84.06 & 77.27 & 0.00007 & 22.72 & 80.52 & 0.429 \\
\hline \multicolumn{8}{|c|}{ (c) Brazil's forest } \\
\hline MSR-U-net & 99.99 & 86.14 & 87.15 & 0.001 & 12.85 & 86.64 & 0.429 \\
\hline Fire-net & 99.99 & 95.98 & 98.04 & 0.0004 & 1.95 & 97.00 & 0.429 \\
\hline \multicolumn{8}{|l|}{ (d) Chernobyl } \\
\hline MSR-U-net & 99.99 & 86.14 & 87.15 & 0.004 & 15.45 & 81.96 & 0.429 \\
\hline Fire-net & 99.99 & 95.98 & 98.04 & 0.0006 & 4.58 & 97.24 & 0.429 \\
\hline
\end{tabular}

TABle 3: Accuracy assessment of active fire detection (Comparison of five common Classification methods).

\begin{tabular}{|c|c|c|c|c|c|c|c|}
\hline Method & OA (\%) & Precision (\%) & Recall (\%) & FPR (\%) & $\mathrm{MD}(\%)$ & F1-score $(\%)$ & $\mathrm{KC}$ \\
\hline \multicolumn{8}{|c|}{ (a) Australian's Forest } \\
\hline MLP & 99.02 & 0.00 & 0.00 & 0.009 & 100 & 0.00 & 0.00 \\
\hline $\mathrm{KNN}$ & 97.13 & 25.13 & 99.63 & 2.88 & 0.37 & 40.14 & 0.391 \\
\hline $\mathrm{RF}$ & 96.29 & 20.66 & 100 & 3.73 & 0.00 & 34.24 & 0.331 \\
\hline SVM & 99.03 & 14.28 & 0.01 & 0.001 & 99.98 & 0.03 & 0.03 \\
\hline XGBOOST & 96.29 & 20.66 & 100 & 3.73 & 0 & 34.24 & 0.331 \\
\hline \multicolumn{8}{|c|}{ (b) Central Africa's Forest in 2018-12-19 } \\
\hline MLP & 99.48 & 0.00 & 0.00 & 0.51 & 100 & 0.00 & 0.1964 \\
\hline $\mathrm{KNN}$ & 99.99 & 79.95 & 70.20 & 0.01 & 29.79 & 72.96 & 0.4293 \\
\hline $\mathrm{RF}$ & 99.99 & 57.47 & 75.75 & 0.02 & 24.24 & 65.35 & 0.4293 \\
\hline SVM & 99.99 & 0 & 0 & 0 & 100 & 0 & 0.4293 \\
\hline XGBOOST & 99.99 & 57.47 & 75.75 & 0.02 & 24.24 & 65.35 & 0.4293 \\
\hline \multicolumn{8}{|c|}{ (c) Brazil's Forest } \\
\hline MLP & 99.64 & 0.002 & 0.08 & 0.34 & 99.91 & 0.34 & 0.1426 \\
\hline $\mathrm{KNN}$ & 99.99 & 60.16 & 91.77 & 0.006 & 8.22 & 0.006 & 0.4294 \\
\hline $\mathrm{RF}$ & 99.98 & 49.93 & 98.19 & 0.011 & 1.80 & 66.20 & 0.4294 \\
\hline SVM & 99.98 & 50.00 & 0.02 & 0.00 & 99.97 & 0.04 & 0.4292 \\
\hline XGBOOST & 99.98 & 49.93 & 98.19 & 0.011 & 1.80 & 66.20 & 0.4294 \\
\hline \multicolumn{8}{|c|}{ (d) Chernobyl } \\
\hline MLP & 99.99 & 0 & 0 & 1.27 & 100 & 0 & 0.2705 \\
\hline $\mathrm{KNN}$ & 99.99 & 83.84 & 75.60 & 0.00 & 24.39 & 79.51 & 0.4293 \\
\hline $\mathrm{RF}$ & 99.99 & 70.35 & 91.17 & 0.00 & 8.83 & 79.42 & 0.4293 \\
\hline SVM & 99.99 & 0 & 0 & 0 & 100 & 0 & 0.4293 \\
\hline MLP & 99.99 & 70.35 & 91.17 & 0.00 & 8.83 & 79.42 & 0.4293 \\
\hline
\end{tabular}

this metric, Fire-Net had a score of more than $93 \%$ compared to MSR-U-Net at $67 \%$. The high rate of F1-Scores expresses the model has either the high believable and high ability in prediction of TP pixels. The OA index is one of the most common factors in quantity assessment. This index associates the relation among $\mathrm{TN}$ and $\mathrm{TP}$ pixels with all pixels. The OA proposed Fire-Net algorithm is more than 99\% in all datasets. This subject shows the proposed method has high performance in prediction $\mathrm{TP}$ and true negative
(TN) pixels. The $\mathrm{KC}$ measures the reliability of model in classification that mainly, the proposed method was provided the moderated level.

MD and FPR are two metrics that should be low for effective active fire detection. This is especially true for MD because undetected fire pixels are more costly than labeling false detections. Our results show that Fire-Net's average MD is at $8 \%$ with an FPR of $0.0001 \%$. MSR-U-Net on the other hand had an average of MD rate more than $14 \%$ with 


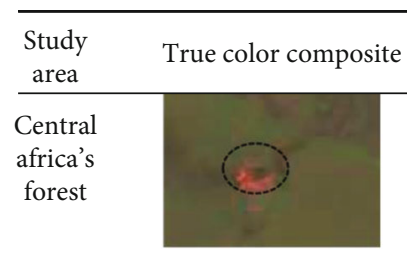

(a)

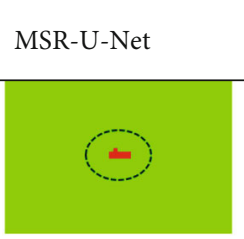

(b)
Proposed method

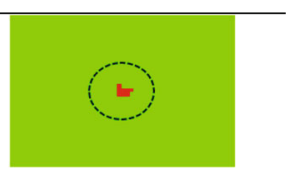

(c)

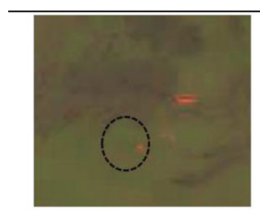

(d)

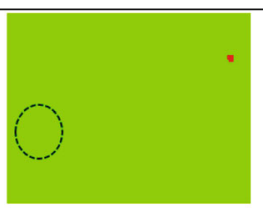

(e)

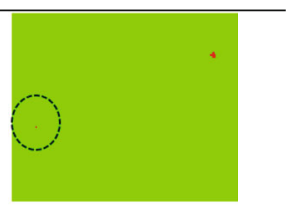

(f)

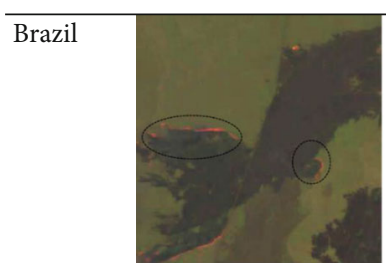

(g)

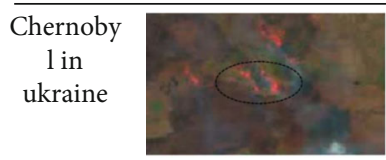

(j)

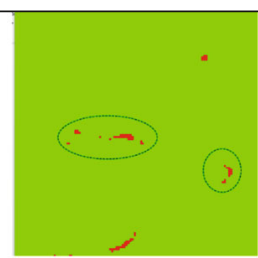

(h)

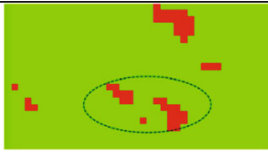

$(\mathrm{k})$

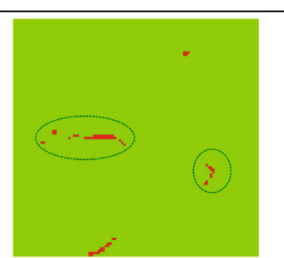

(i)

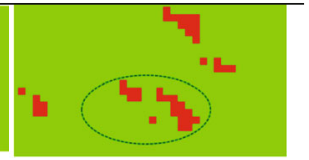

$(\mathrm{m})$

Figure 7: The zoom active fire area and red and green are fire and non-fire, respectively.

TABLE 4: Comparison of performance proposed Fire-Net algorithm with other fire detection methods.

\begin{tabular}{|c|c|c|c|}
\hline & Index & Method & Dataset \\
\hline Saeed, et al. [40] & OA: $99(\%)$ & Deep learning based & Close rage dataset \\
\hline Jang, Kang, Im, Lee, Yoon and Kim [17] & $\begin{array}{l}\text { Precision: } \\
\quad 93.08\end{array}$ & RF and threshold based & $\begin{array}{c}\text { Himawari-8 geostationary satellite } \\
\text { data }\end{array}$ \\
\hline Jiao, Zhang, Xin, Mu, Yi, Liu and Liu [18] & $\begin{array}{l}\text { Precision: } 83 \\
\text { FPR: } 3.2\end{array}$ & $\begin{array}{l}\text { Deep learning } \\
\text { (YOLOv3) }\end{array}$ & UAV dataset \\
\hline $\begin{array}{l}\text { Schroeder, Oliva, Giglio, Quayle, Lorenz and Morelli } \\
{[14]}\end{array}$ & FPR: 0.2 & Thresholding based & Landsat 8 \\
\hline Lin, Chen, Li, Yu, Jia, Zhang and Liang [23] & $\begin{array}{l}\text { OA: } 54 \\
\text { MD: } 78\end{array}$ & Contextual based & FengYun-2G S-VISSR data \\
\hline Proposed fire-net & $\begin{array}{c}\text { Precision: } \\
93.49 \\
\text { FPR: } 0.0001 \\
\text { OA: } 99.98\end{array}$ & Deep learning based & Landsat 8 \\
\hline
\end{tabular}

a mean FPR of 1\%. Essentially, precision, recall, and the F1Scores significantly improved by the Fire-Net algorithm within all study areas. From the valuations, the 5 machine learning algorithms showed high performance, mainly in mapping non-fire areas. They however failed to map active fires. In comparison to the deep networks (especially to Fire-Net), all 5 machine learning methods had lower success rates. Fire-Net showed the highest precision for the active fire detection task. Recently, more research has been done for active fire detection based on RS imagery. Table 4 presents the obtained results by other active fire detection methods.

There are some products for active fire detection as globally that they mainly are generated by MODIS, VIIRIS (Visible Infrared Imaging Radiometer Suite), and NOAA (National Oceanic and Atmospheric Administration) 
sensors. Oliva and Schroeder [52] investigated the active fire product based on VIIRS dataset by comparing the Landsat-8 dataset. The result of mentioned study shows the accuracy of this product depend on size fires as the accuracy of product covers ranges $7 \%$ through $100 \%$. Furthermore, Giglio et al. [53] had investigated the performance of collection six MODIS active fire products. The numerical results had shown the FPR rate of this product is nearby $2.4 \%$.

One of most important issue of deep learning method is balancing dataset. The fire areas cover very small areas while the non-fire areas are dominant in the scene. Therefore, for solving this issue we introduced novel loss function. This loss function combines dice-loss and weighted-binary cross-entropy.

5.2. Feature Extraction. More active fire detection methods focus on spectral features and contextual information [30]. The ability of deep learning methods for feature extraction have been proven by many researches [12, 40, 42, 43, 54]. The deep learning methods are able to detect deep features containing various spectral/spatial features. The quality and quantity of active fire methods based on manual hand crafted features is the main challenge in traditional active fire detection methods. The deep learning methods can extract deep informative features in an automatic manner. It is worth noting that the proposed method used only four coarse bands (Red, Green, Blue, and thermal). These bands are the most common bands and widely available data in most RS satellite imagery. Therefore, this methodology can be deployed by other satellites with higher temporal resolution in active fire detection and forest management for real time and continuous fire monitoring.

5.3. Transferability. Most of the proposed active fire detection methods are based on thresholding [21, 24, 55]. Due to different environmental conditions and diversity in forest layouts, it is impractical to fix constant threshold values for decision making. From deep learning point of view, both the methods are based on deep learning, which does not require hardcoding threshold values. Instead, during the training step, each network discovers the best weights connecting each node through an optimization process. The transferability is clearly shown with high accuracy for all regions.

\section{Conclusion}

Early detection of active forest fires is critical to determine the starting point of the fire for effective emergency responses. In this study, active fire detection was performed on a medium spatial resolution dataset (Landsat- 8 imagery) where the extent of active fires was very low. A deep CNN (Fire-Net) was proposed in this work to detect active forest fires in various regions. Specifically, the USA and Australia regions were used to train the network whereas testing was done for Africa, Brazil, Ukraine, Australia (the parts was not involved in training) regions. The results depicted a high transferability of the proposed method. Then, the Fire-Net was compared with another state-of-the-art deep network, i.e., MSR-U-Net and other common machine learning algorithms. The results for active fire detection were qualitatively and quantitatively assessed. The performance evaluations showed there was a trade-off between active fire and nonactive fires detection. Due to the extent of active fires in small areas, most of the machine learning algorithms could not detect active fires. The high accuracies measured by $\mathrm{OA}$ index for these algorithms, were mostly for non-fire zones. The other indices namely precision, $\mathrm{KC}$, and F1score for these models were low for the detection of active fires. In contrast, the proposed Fire-Net method showed high efficacy for both active and non-active fire detection. Here, small active fires were detected with high accuracy and low miss detection rates. The efficiency of Fire-Net originated in its architecture and convolution layers structure enabling high level and informative features extraction. Experimental results indicate that Fire-Net: (1) has higher accuracy, (2) obtains higher sensitivity to small active fires, (3) the proposed method can be applied as real time processing due to high transferability. Future work will be based on using geostationary satellite imagery for rapid monitoring of active fires to provides high temporal resolution for fast monitoring in a larger scale.

\section{Data Availability}

The data used to support the findings of this study are available from the corresponding author upon request.

\section{Conflicts of Interest}

The author(s) declare(s) that they have no conflicts of interest.

\section{References}

[1] D. Oom and J. M. C. Pereira, "Exploratory spatial data analysis of global MODIS active fire data," International Journal of Applied Earth Observation and Geoinformation, vol. 21, pp. 326-340, 2013.

[2] K. Ahmadi, B. Kalantar, V. Saeidi, E. K. G. Harandi, S. Janizadeh, and N. Ueda, "Comparison of machine learning methods for mapping the stand characteristics of temperate forests using multi-spectral sentinel-2 data," Remote Sensing, vol. 12, no. 18, p. 3019, 2020.

[3] B. Kalantar, N. Ueda, V. Saeidi et al., "Deep neural network utilizing remote sensing datasets for flood Hazard susceptibility mapping in Brisbane, Australia," Remote Sensing, vol. 13, no. 13, p. 2638, 2021.

[4] M. J. Wooster, W. Xu, and T. Nightingale, "Sentinel-3 SLSTR active fire detection and FRP product: pre-launch algorithm development and performance evaluation using MODIS and ASTER datasets," Remote Sensing of Environment, vol. 120, pp. 236-254, 2012.

[5] H. A. H. Al-Najjar and B. Pradhan, "Spatial landslide susceptibility assessment using machine learning techniques assisted by additional data created with generative adversarial networks," Geoscience Frontiers, vol. 12, no. 2, pp. 625-637, 2021.

[6] B. Kalantar, N. Ueda, M. O. Idrees, S. Janizadeh, K. Ahmadi, and F. Shabani, "Forest fire susceptibility prediction based on 
machine learning models with resampling algorithms on remote sensing data," Remote Sensing, vol. 12, no. 22, 2020.

[7] S. T. Seydi, M. Akhoondzadeh, M. Amani, and S. Mahdavi, "Wildfire Damage Assessment over Australia Using Sentinel2 Imagery and MODIS Land Cover Product within the Google Earth Engine Cloud Platform," Remote Sensing, vol. 13, no. 2, p. 220, 2021.

[8] M. S. Tehrany, H. Özener, B. Kalantar et al., "Application of an ensemble statistical approach in spatial predictions of bushfire probability and risk mapping," Journal of Sensors, vol. 2021, Article ID 6638241, 31 pages, 2021.

[9] C. Vancutsem, F. Achard, J. F. Pekel et al., "Long-term (19902019) monitoring of forest cover changes in the humid tropics," Science Advances, vol. 7, no. 10, pp. 1-22, 2021.

[10] I. G. Hendel and G. M. Ross, "Efficacy of remote sensing in early Forest fire detection: a thermal sensor comparison," Canadian Journal of Remote Sensing, vol. 46, no. 4, pp. 414428, 2020.

[11] C. A. Varotsos, V. F. Krapivin, and F. A. Mkrtchyan, “A new passive microwave tool for operational forest fires detection: a case study of siberia in 2019," Remote Sensing, vol. 12, no. 5, p. 835, 2020.

[12] B. Pradhan, H. A. H. Al-Najjar, M. I. Sameen, I. Tsang, and A. M. Alamri, "Unseen land cover classification from HighResolution orthophotos using integration of zero-shot learning and convolutional neural networks," Remote Sensing, vol. 12, no. 10, 2020.

[13] M. B. A. Gibril, B. Kalantar, R. Al-Ruzouq et al., "Mapping heterogeneous urban landscapes from the fusion of digital surface model and unmanned aerial vehicle-based images using adaptive multiscale image segmentation and classification," Remote Sensing, vol. 12, no. 7, p. 1081, 2020.

[14] M. Hasanlou, R. Shah-Hosseini, S. T. Seydi, S. Karimzadeh, and M. Matsuoka, "Earthquake damage region detection by multitemporal coherence map analysis of radar and multispectral imagery," Remote Sensing, vol. 13, no. 6, p. 1195, 2021.

[15] M. Hasanlou and S. T. Seydi, "use of multispectral and hyperspectral satellite imagery for monitoring Waterbodies and Wetlands," Southern Iraq's Marshes, vol. 36, pp. 155-181, 2021.

[16] J. E. Pereira-Pires, V. Aubard, R. A. Ribeiro, J. M. Fonseca, J. M. N. Silva, and A. Mora, "Semi-automatic methodology for fire break maintenance operations detection with sentinel-2 imagery and artificial neural network," Remote Sensing, vol. 12, no. 6, p. 909, 2020.

[17] S. Mukai, I. Sano, and M. Nakata, "Improved algorithms for remote sensing-based aerosol retrieval during extreme biomass burning events," Atmosphere, vol. 12, no. 3, p. 403, 2021.

[18] A. A. Floutsi, H. Baars, M. Radenz et al., "Advection of biomass burning aerosols towards the southern hemispheric midlatitude station of Punta Arenas as observed with multiwavelength polarization raman lidar," Remote Sensing, vol. 13, no. $1,2021$.

[19] S. Ye, J. Rogan, Z. Zhu, and J. R. Eastman, “A near-real-time approach for monitoring forest disturbance using Landsat time series: stochastic continuous change detection," Remote Sensing of Environment, vol. 252, p. 112167, 2021.

[20] J. Reiche, A. Mullissa, B. Slagter et al., "Forest disturbance alerts for the Congo Basin using Sentinel-1," Environmental Research Letters, vol. 16, no. 2, p. doi:10.1088/1748-9326/ abd0a8, 2021.
[21] W. Schroeder, P. Oliva, L. Giglio, B. Quayle, E. Lorenz, and F. Morelli, "Active fire detection using Landsat-8/OLI data," Remote Sensing of Environment, vol. 185, pp. 210-220, 2016.

[22] H. Cruz, M. Eckert, J. Meneses, and J. F. Martínez, "Efficient forest fire detection index for application in unmanned aerial systems (UASs)," Sensors, vol. 16, no. 6, 2016.

[23] W. Schroeder, P. Oliva, L. Giglio, and I. A. Csiszar, "The New VIIRS $375 \mathrm{~m}$ active fire detection data product: Algorithm description and initial assessment," Remote Sensing of Environment, vol. 143, pp. 85-96, 2014.

[24] E. Jang, Y. Kang, J. Im, D. W. Lee, J. Yoon, and S. K. Kim, "Detection and monitoring of forest fires using Himawari-8 geostationary satellite data in South Korea," Remote Sensing, vol. 11, no. 3, p. 271, 2019.

[25] Z. Jiao, Y. Zhang, J. Xin et al., “A deep learning based forest fire detection approach using uav and yolov3," in 2019 1st International Conference on Industrial Artificial Intelligence (IAI), pp. 1-5, Shenyang, China, 2019.

[26] C. Yuan, Z. Liu, and Y. Zhang, "Aerial images-based Forest fire detection for firefighting using optical remote sensing techniques and unmanned aerial vehicles," Journal of Intelligent \& Robotic Systems, vol. 88, no. 2-4, pp. 635-654, 2017.

[27] M. Amraoui, C. C. DaCamara, and J. M. C. Pereira, "Detection and monitoring of African vegetation fires using MSG-SEVIRI imagery," Remote Sensing of Environment, vol. 114, no. 5, pp. 1038-1052, 2010.

[28] Y. Chen, Y. Zhang, J. Xin et al., "UAV image-based forest fire detection approach using convolutional neural network," in 2019 14th IEEE Conference on Industrial Electronics and Applications (ICIEA), pp. 2118-2123, Xi'an, China, 2019.

[29] M. Gargiulo, D. A. G. Dell' Aglio, A. Iodice, D. Riccio, and G. Ruello, "A CNN-based super-resolution technique for active fire detection on Sentinel-2 data," in 2019 PhotonIcs \& Electromagnetics Research Symposium - Spring (PIERS-Spring), pp. 418-426, Rome, Italy, 2019.

[30] Z. Lin, F. Chen, B. Li et al., "A contextual and multitemporal active-fire detection algorithm based on FengYun-2G SVISSR data," IEEE Transactions on Geoscience and Remote Sensing, vol. 57, no. 11, pp. 8840-8852, 2019.

[31] T. Zhang, M. J. Wooster, and W. Xu, “Approaches for synergistically exploiting VIIRS I- and M-band data in regional active fire detection and FRP assessment: a demonstration with respect to agricultural residue burning in eastern China," Remote Sensing of Environment, vol. 198, pp. 407-424, 2017.

[32] J. San-Miguel-Ayanz, N. Ravail, V. Kelha, and A. Ollero, "Active fire detection for fire emergency management: potential and limitations for the operational use of remote sensing," Natural Hazards, vol. 35, no. 3, pp. 361-376, 2005.

[33] H. A. Al-Najjar, B. Kalantar, B. Pradhan et al., "Land cover classification from fused DSM and UAV images using convolutional neural networks," Remote Sensing, vol. 11, no. 12, p. 1461, 2019.

[34] "National Aeronautics and Space Administration NASA Official: Brian Dunbar Available online," September 2021, https:// www.nasa.gov/image-feature/goddard.

[35] "National Aeronautics and Space Administration NASA Official Available online," September 2021, https://www.nasa.gov/.

[36] F. H. Maskouni and S. T. Seydi, "Forest burned area mapping using bi-temporal Sentinel-2 imagery based on a convolutional neural Network: Case Study in Golestan Forest," Engineering Proceedings, vol. 10, no. 1, pp. 6-11, 2021. 
[37] A. Veit, M. J. Wilber, and S. Belongie, "Residual networks behave like ensembles of relatively shallow networks," Advances in Neural Information Processing Systems, vol. 29, 2016.

[38] F. Chollet, "Xception: Deep learning with depthwise separable convolutions," in Proceedings of the IEEE Conference on Computer Vision and Pattern Recognition (CVPR), pp. 1251-1258, Honolulu, Hawaii, USA, 2017.

[39] S. T. Seydi and M. Hasanlou, "Binary hyperspectral change detection based on 3D convolution deep learning," International Archives of the Photogrammetry, Remote Sensing \& Spatial Information Sciences, vol. XLIII-B3-2020, pp. 1629-1633, 2020.

[40] S. T. Seydi and H. Rastiveis, "A deep learning framework for roads network damage assessment using post-earthquake lidar data," International Archives of the Photogrammetry, Remote Sensing \& Spatial Information Sciences, vol. XLII-4/W18, pp. 955-961, 2019.

[41] S. T. Seydi, M. Hasanlou, and M. Amani, "A new end-to-end multi-dimensional CNN framework for land cover/land use change detection in multi-source remote sensing datasets," Remote Sensing, vol. 12, no. 12, p. 2010, 2020.

[42] S. Nah, T. H. Kim, and K. M. Lee, "Deep multi-scale convolutional neural network for dynamic scene deblurring," in Proceedings of the IEEE Conference on Computer Vision and Pattern Recognition (CVPR), pp. 257-265, Honolulu, Hawaii, USA, 2017.

[43] K. He, X. Zhang, S. Ren, and J. Sun, "Deep residual learning for image recognition," in Proceedings of the IEEE conference on computer vision and pattern recognition, pp. 770-778, Las Vegas, NV, USA, 2016.

[44] A. G. Howard, M. Zhu, B. Chen et al., "MobileNets: Efficient Convolutional Neural Networks for Mobile Vision Applications," http://arxiv.org/abs/1704.04861.

[45] M. Sandler, A. Howard, M. Zhu, A. Zhmoginov, and L. C. Chen, "MobileNetV2: inverted residuals and linear bottlenecks," in Proceedings IEEE Conference on Computer Vision and Pattern Recognition, pp. 4510-4520, 2018.

[46] J. Wang, S. Feng, Y. Cheng, and N. Al-Nabhan, "Survey on the loss function of deep learning in face recognition," Journal of Information Hiding and Privacy Protection, vol. 3, no. 1, pp. 29-45, 2021.

[47] K. C. L. Wong, M. Moradi, H. Tang, and T. Syeda-Mahmood, "3D segmentation with exponential logarithmic loss for highly unbalanced object sizes," in International Conference on Medical Image Computing and Computer-Assisted Intervention, pp. 612-619, Springer, 2018.

[48] D. P. Kingma and J. L. Ba, “Adam: A method for stochastic optimization," http://arxiv.org/abs/1412.6980.

[49] Z. Liu, H. Wang, L. Weng, and Y. Yang, "Ship rotated bounding box space for ship extraction from high-resolution optical satellite images with complex backgrounds," IEEE Geoscience and Remote Sensing Letters, vol. 13, no. 8, pp. 1074-1078, 2016.

[50] L. Bao, Z. Yang, S. Wang, D. Bai, and J. Lee, "Real image denoising based on multi-scale residual dense block and cascaded U-net with block-connection," in Proceedings of the IEEE/CVF Conference on Computer Vision and Pattern Recognition (CVPR) Workshops, pp. 1823-1831, 2020.

[51] H. Nian, P. Cheng, and Z. Q. Zhu, "Independent operation of DFIG-based WECS using resonant feedback compensators under unbalanced grid voltage conditions," IEEE Transactions on Power Electronics, vol. 30, no. 7, pp. 3650-3661, 2015.

[52] P. Oliva and W. Schroeder, "Assessment of VIIRS $375 \mathrm{~m}$ active fire detection product for direct burned area mapping," Remote Sensing of Environment, vol. 160, pp. 144-155, 2015.

[53] L. Giglio, W. Schroeder, and C. O. Justice, "The collection 6 MODIS active fire detection algorithm and fire products," Remote Sensing of Environment, vol. 178, pp. 31-41, 2016.

[54] B. Kalantar, N. Ueda, H. A. H. Al-Najjar, and A. A. Halin, "Assessment of convolutional neural network architectures for earthquake-induced building damage detection based on Pre- and post-event orthophoto images," Remote Sensing, vol. 12, no. 21, p. 3529, 2020.

[55] A. A. A. Alkhatib, "A review on forest fire detection techniques," International Journal of Distributed Sensor Networks, vol. 10, no. 3, 2014. 